\title{
Discussion to papers of Dr Dollfus et al., J. Claus-Walker et al., Dr Ohry et al. and Dr B. A. Green
}

\author{
Chairman: Professor PAESLACK
}

\begin{abstract}
UNKNown voICE (USA or Canada). Is this time related, I didn't hear when you were receiving your patients.

DR P. Dollfus (France). We see, I would say about 90 per cent about half an hour to an hour after the accident and we start subcutaneous heparine on a 24 -hour basis, that is, three times a day right from the first day. That is from the first day we see them, practically one hour after the accident in one of the resuscitation units here, let's say for about 80 per cent of our patients. Then with the resuscitation specialists we start these people, unless they have an associated head injury. If they have had an associated head injury we wait a little bit, but if they don't we start them practically 3 or 4 hours after the accident.
\end{abstract}

DR GeIsLer (Canada). The reason why I asked the question we are very concerned about the fact that we are not treating enough patients, as you pointed out. However, we have a very carefully developed special project where we are looking at spinal cord injuries in deep venous thrombosis and patients who have complete neurological injury and no involvement of the lower extremities and we are using the pre-selective studies that you have just reported. The incidence of deep venous thrombosis in our spinal cord patients with that type of criteria was 90 per cent following trauma. Many of these patients come in with multiple trauma and it is a very difficult decision as to how to treat multiple trauma victims on heparine and I would like you to answer that.

DR Dollfus (France). We adapt. We start by using fairly low doses what we call 0.25 which is 2,500 international units every 8 hours, not 12 hours but every 8 hours, that is, if they do not have a head injury. If they have an associated head injury we sometimes wait one day or two days before starting them. We have had no complications related really to this method only as I mentioned three cases had a haematuria, one case which will be published in Paraplegia had proconvertin factor deficiency or a Stuart factor deficiency so we start these patients practically immediately after. Now Watson has shown clearly that it has diminished at least clinically the number of thrombosis and emboli. We have had in our series probably one pulmonary emboli we can account for but we really were mistaken by all these blood changes which were congenital. In fact this is where we lost ourselves, because we went very low. We calculated according to the ITP went down to one or two which is very low and we didn't really know what was going to happen. We couldn't really understand why we had to adapt so low doses for this patient. This patient had a pulmonary emboli and after that he was treated with a massive dose of subcutaneous Heparin and he had haemorrhaged breathing which with a paraparesis, hemiparesis on one side. He recovered and just as he left, he was walking with two sticks, just as he left the Centre one of the glass doors hit him, he fell down and broke his hip, so we had him again, and this time he was more hyper-coagulable than hypo-coagulable as he was in the first instance. We just can't explain why. But in the majority of the cases, we see these cases very early, and we do start sub-cutaneous Heparin extremely early if we can, even if the patient is bleeding, even if he has a haemothorax. You can see a haemothorax, you can look at the X-ray but the pulmonary emboli you cannot see it. I think we will go on using this method for the time being until we find something better to prevent our patients from having thrombosis and embolus, but perhaps Dr Henon who is a blood specialist could have a word on that.

PROF. PAESLACK. I'm sorry gentlemen but these are proferred papers only. I think it would be interesting to discuss this problem for some hours but we have to be on time and may I ask now Dr Walker and Dr Ohry to give their papers. 
UNKNOWN SPEAKER. Dr Claus Walker, I am fascinated by your findings because we had a patient lately who had hypercalcemia, of course a tetraplegic, a recently injured boy about two months after the injury, and he lost a lot of weight. He fell down. He was I $4 \frac{1}{2}$ years old and he was about $6 \mathrm{ft}$ tall and came down to a weight of 38 kilos and the physician judged that he needed intravenous alimentation, and when they gave him this and he lost up to 2 grams of calcium a day in the urine, and he was given intravenous fluid without any calcium. I went to the literature and I found out that a patient undergoing hyper-alimentation was losing a lot of zinc and that showed a zinc toxity. Maybe we can get together for investigating what it does, what we should add to the intravenous fluid.

DR OHRY (Israel). I don't think I have much to add to these remarks.

Dr GeISLER (Canada). Dr Ohry how did you determine that your patient had osteoporosis?

DR OHRY. I presumed that spinal cord injured patients, paraplegic and tetraplegic, confined to bed during the immobilisation periods are osteoporotic that it is shown in X-ray films.

Prof. Paeslack. Now we have the next paper, it is by Dr Green.

SIR GeORge Bedbrook (Australia). I would like to ask Dr Green a question as to whether his work study activities were devised in comparison between so-called kinetic nursing and conventional nursing, because quite frankly until such work studies were carefully undertaken by competent people I wouldn't really believe that kinetic nursing is any better or any worse than ordinary nursing. Secondly, if his mortality is 7 to 105 mine is 2 to IOo. If he has pulmonary problems, we don't get them. Urinary stones we haven't seen for a long long time, autonomic hyperreflexia we don't see, gastro-intestinal haemorrhage I can't remember a patient with that. Lack of function, I haven't seen one neurological dysfunction and one neurological aggravation for a long time. My stability rate is 88 per cent and 96 per cent stability, passive movements can be done on ordinary conventional beds, and I don't really think that the reason for his kinetic activity. As far as the Keane's bed is concerned, we had one and this was a very expensive investment as far as we were concerned in Western Australia, it's regretfully in the basement because our nurses won't use it.

DR GREEN (U.S.A.). Unfortunately I didn't hear all of your points but I am quite impressed by your lack of any complications, I think you should publish your series. As far as our list of mobility and mortality I have to remind you that the group I presented were 90 per cent, were ten to one quadriplegics to paraplegics. I am in a major metropolitan problem centre where nobody just comes in with a broken neck, they also have multiple injuries, the majority of patients, and I am quite interested, I would be happy to compare apples to apples but not apples to oranges. So I would like to run a patient-topatient comparison between your multi-trauma acutely injured patients with cervical injuries and mine and then I think we could discuss it better. But right now, I don't think it is a proper way to compare because I don't think we are talking about the same thing. You have never had a pulmonary embolism in your Unit, is that correct? O.K. well we have never had a pulmonary embolism on a patient who is kinetically acute and he could never have been on heparin or aspirin just for one example.

SIR Ludwig GutTMANN (G.B.). I don't think you have answered the question. You were clearly asked if you have made comparative investigations. You obviously haven't done it. Kinetic nursing has by no means started with Dr Keane's Rota-Rest Mark I. Kinetic Nursing as opposed to the traditional nursing of traumatic spinal cord injuries in recumbency was started during the last stages of World War II together with Dynamic Physiotherapy as the most important components of the comprehensive management of spinal cord injuries, pioneered at Stoke Mandeville. The regular manual turning of the patient every 2 hours day and night was the Kinetic Nursing at Stoke Mandeville which proved so very successful in healing and preventing sores, stagnation of urine, with all the consequences of ascending urinary infection, etc. This manual kinetic nursing was later replaced by the Stoke Mandeville-Egerton electrically controlled turning and tilting bed with a special head traction unit for traumatic tetraplegics. Your criticism of the Stoke Mandeville bed obviously lacks entirely proper comparative studies. In any case, Kinetic 
Nursing has for many years been accepted as the ordinary and conventional nursing procedure in the management and care of spinal cord sufferers. Keane's Roto-Rest so much advertised by you has been discussed previously in this Society and met with criticism. In this respect Sir George Bedbrook's criticism made today is quite significant.

DR GREEN. I'm sorry. You obviously weren't listening to the first part of the paper. We have made historical comparisons to other series and percentages and as you will see, in the publication, we reviewed the series in the literature as far as pulmonary emboli and respiratory complications, so there are historical comparisons. As far as within our own Centre, doing a double guide perspective study, we haven't done that because we didn't feel that we could justify randomising patients because of our experience early on with the Rota-Rest being such a positive one. As far as mechanically not working we had the same trouble you did. The early models had a lot of mechanical problems and the differences today are like night and day, we are not having these problems any more as we used to have, but they do exist.

SIR GEORge BedBrook (Australia). Mr Chairman, I must answer this. I don't think Dr Green understands what I mean by a work study. I want to know the percentage of nurse time and the percentage of time by technicians and the percentage of doctor time, the percentage time taken to do a dressing and as for washing or not changing gowns, I disagree with both the speakers, there is always time for that. I have work studied these. You can do an ordinary turn in 2 minutes, you can wash in 30 seconds and you can change a gown in 15 seconds.

DR GREEN. We do wash our patients in America too. As a matter of fact, our patients are kinetically nursed about 20 out of 24 hours on average and our patients receive a daily bath, are given physiotherapy, occupational therapy, respiratory therapy. They eat with the beds stopped in many cases. So it is not incontinuous but we do stop and we limit the time. I made a statement near the end that we did do a time study and we found that kinetic nursing doesn't reduce the amount of time that it takes for nursing care but just allows them to change the focus of the care. They don't have to spend their time lifting and turning, instead they can spend their time on what we consider more important things. 